\title{
An outbreak of staphylococcal food poisoning in a nursing hostel, Mysore, Karnataka
}

Objectives: To know the clinical profile of the students affected due to food poisoning, the possible source of infection and the causative agent for food poisoning. Background: Food-borne diseases due to microbial contamination of food, represent serious threat to the health of millions of people henceforth, we investigated into the outbreak of food poisoning in a nursing hostel of urban Mysore. Materials and Methods: A descriptive study was conducted using semi-structured questionnaire to gather information on regarding time and type of food item consumed, onset of symptoms, presenting symptoms, medical care provided and time taken for recovery. A case for the outbreak was defined as any person who had consumed lunch on 9/12/12 from the implicated kitchen and had experienced vomiting or diarrhea or pain abdomen or all the three symptoms within 24 hours of eating lunch. Results: Out of 171 inmates, 46 people suffered from food poisoning within a period of 3 days,thus leading to the attack rate of $26.9 \%$.Commonest symptom reported among the students was pain abdomen $(26.9 \%)$ followed by diarrhea. Highest risk of food poisoning was associated with consumption of "Kerala matta rice" This association was statistically significant with relative risk of 31.49 (95\% Cl 10.82-95.6). Samples of "Kerala matta rice" was subjected to bacteriological analysis, which showed colonies of coagulase-positive Staphylococcus aureus. Conclusions: In the present outbreak, the results of investigation revealed that 'Kerala matta rice' was the food item responsible for occurrence of food poisoning due to Staphylococcus. Environmental factors that could have played an important role in bacterial proliferation and enterotoxin production are manual handling of cooked rice by the food handlers and storage of the rice at room temperature for a long duration between time of preparation and consumption.

Key words: Hostel, outbreak, staphylococcal food poisoning

Gowdara Shankarappa Vijaykumar $^{1}$

Departments of Community Medicine and ${ }^{1}$ Microbiology, JSS Medical College, JSS University,

Mysore, Karnataka, India

Address for the Correspondence: Dr. Madhu B,

Department of Community Medicine, JSS Medical College, JSS University, Mysore - 570015,

Karnataka, India.

E-mail: drmadhusri@gmail.com

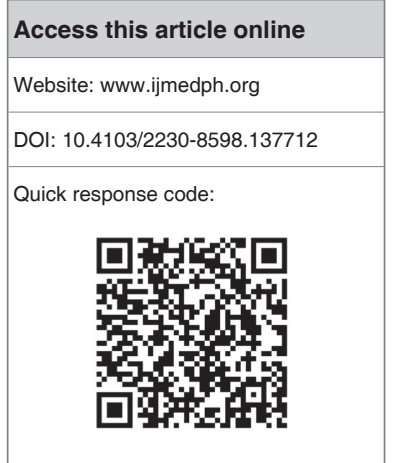

\section{INTRODUCTION}

Food safety is an essential public health issue in all countries. Food-borne diseases due to microbial pathogens, bio-toxins, and chemical contaminants in food represent serious threat to the health of millions of people. Serious outbreaks of food borne disease have been documented on every continent in the past decades, illustrating both the public health and social significance of these diseases. $^{[1]}$

Hostel inmates of various educational institutions constitute a high risk group for food poisoning. This study describes an outbreak of food poisoning that occurred among inmates of a nursing hostel in Mysore. The investigation into the outbreak of food poisoning was conducted to know the clinical profile of the patients affected, to find out the possible source of infection and the causative organism, to end the progression of the outbreak and to prevent any future occurrences of outbreak of food poisoning.

\section{MATERIALS AND METHODS}

The study was of descriptive type. Study was undertaken among all inmates of nursing hostel who had consumed food from the same catering services between 9/12/2012 and 12/12/2012 irrespective of presence or absence of symptoms. Data pertaining to the outbreak was collected using a pre-formed semi-structured questionnaire from the inmates of the hostel. It included information regarding time 
and type of food item consumed, onset of symptoms, presenting symptoms, medical care provided and time taken for recovery.

Assessment of the clinico-epidemiological profile of the persons affected suggested that the lunch provided on 9/12/12 was responsible for the outbreak. A case for the outbreak was defined as, any person who had consumed lunch on 9/12/12 from the implicated kitchen and had experienced vomiting or diarrhea or pain abdomen or all the three symptoms within 24 hours of eating lunch. Diarrhea was defined as three or more loose stools within a 24-hour period. Incubation period was assessed and attack rate was calculated. Relative risk with $95 \%$ confidence interval $(95 \%$ CI) was calculated for each item to assess the association between consumption of individual food items and subsequent illness.

$\mathrm{RR}=\frac{\text { incidence among exposed }}{\text { incidence among non exposed }}$

An environmental survey was undertaken to investigate into the process of food preparation, storage of food, food transportation and place of consumption of food. Sample of food incriminated was collected and subjected to bacteriological culture. Water used for drinking from the water filter in the hostel was also collected and sent for bacteriological examination. Food handlers were screened for health problems. Health education regarding the common sources for food poisoning and measures to prevent the same was given to cooks and food handlers of the catering services.

\section{RESULTS}

There were a total of 171 inmates residing in the nursing hostel, which is located in the urban Mysore. Out of 171 inmates, 46 people suffered from food poisoning within a period of 3 days between $9 / 12 / 2012$ to $12 / 12 / 2012$. Thus leading to the attack rate of $26.9 \%$. Out of 46 affected, only 23 cases reported to the hospital. Out of 23 cases reported, 17 were admitted as inpatient and 6 cases were treated as outpatient. The index case was reported to the hospital within 12 hours of consumption of food. Food for the hostel inmates was not prepared in the hostel kitchen but it was catered by an outside caterer who has been supplying food. All the inmates affected had consumed the same food by the private caterers during the period of outbreak.

The main symptoms reported by affected students are shown in Table 1 . The commonest symptom reported among the patients was pain abdomen $(26.9 \%)$ followed by diarrhea. More than threefourth of the cases had the onset of symptoms within 12 hours of consumption of food. Nearly one-third (36.95\%) of the cases required hospitalization. All cases were discharged within 48 hours of admission. No deaths were reported.

The peak incidence of cases was on 10/12/12 and the curve [Figure 1] shows a gradual increase and the fall of cases after 12/12/12. Median incubation period was found to be 2 days ( 48 hours). Figure 2 shows a decreasing trend in the admission of cases and the maximum number of cases were admitted on 10/12/12. Relative risk was calculated for each food item. Highest risk of food poisoning was associated with consumption of "Kerala matta rice" (See Table 2). This association was statistically significant with relative risk of 31.49 (95\% CI 10.82-95.6).

Samples of "Kerala matta rice" was subjected to bacteriological analysis, which showed colonies of coagulase-positive Staphylococcus aureus (S.aureus) after 24 hours incubation at $37^{\circ} \mathrm{C}$. Water samples collected from the water filter of the hostel premises showed no significant findings.

\section{DISCUSSION}

Staphylococcus aureus are Gram-positive organisms. Some strains of which are capable of producing a highly heat-stable protein toxin that causes illness in humans. Staphylococcal food poisoning is one of the most common food-borne illnesses. ${ }^{[2]}$ Staphylococci exist in air, dust, sewage, water, milk, and food or on food equipment, environmental surfaces, humans, and animals. Humans and animals are the primary reservoirs. ${ }^{[3]}$ Staphylococci are present in the nasal passage, throat, hair and skin of more than 50 percent healthy individuals and are abundant in cuts, pustules, and abscesses. ${ }^{[4]}$ They thrive in protein-rich foods with a high salt content and thrive in the temperature range of $7^{\circ} \mathrm{C}$ to $48^{\circ} \mathrm{C} . .^{[5]}$

\begin{tabular}{lc}
$\begin{array}{l}\text { Table 1: Distribution of patients according to } \\
\text { symptoms }\end{array}$ & $\begin{array}{c}\text { No. of affected } \\
\mathbf{N}=\mathbf{1 7 1}(\%)\end{array}$ \\
\hline Symptoms & $33(19.2)$ \\
& $12(7.01)$ \\
\hline Diarrhea & $46(26.9)$ \\
Vomiting & $16(9.35)$ \\
Pain abdomen & Fever
\end{tabular}

\begin{tabular}{|c|c|c|c|c|c|c|c|c|}
\hline \multirow[t]{2}{*}{ Food item } & \multicolumn{3}{|c|}{ Ate food item } & \multicolumn{3}{|c|}{ Did not eat food item } & \multirow{2}{*}{$\begin{array}{l}\text { Relative } \\
\text { risk }\end{array}$} & \multirow[t]{2}{*}{$95 \% \mathrm{Cl}^{*}$} \\
\hline & III & Not ill & $\%$ ill & III & Not ill & $\%$ ill & & \\
\hline Chapathi & 23 & 96 & 19.3 & 23 & 29 & 44.2 & 0.43 & $0.26-0.69$ \\
\hline Curry & 23 & 96 & 19.3 & 23 & 29 & 44.2 & 0.43 & $0.26-0.69$ \\
\hline $\begin{array}{l}\text { Kerala matta } \\
\text { rice }\end{array}$ & 43 & 10 & 81.1 & 3 & 115 & 2.5 & 32.4 & $10.82-95.6$ \\
\hline Normal rice & 1 & 106 & 0.9 & 45 & 19 & 70.3 & $0.01^{\#}$ & $0.001-0.06$ \\
\hline Sambhar & 44 & 103 & 29.9 & 2 & 22 & 8.3 & 3.6 & $1.33-9.67$ \\
\hline Curd & 3 & 109 & 2.6 & 43 & 16 & 72.8 & 0.03 & $0.009-0.091$ \\
\hline
\end{tabular}




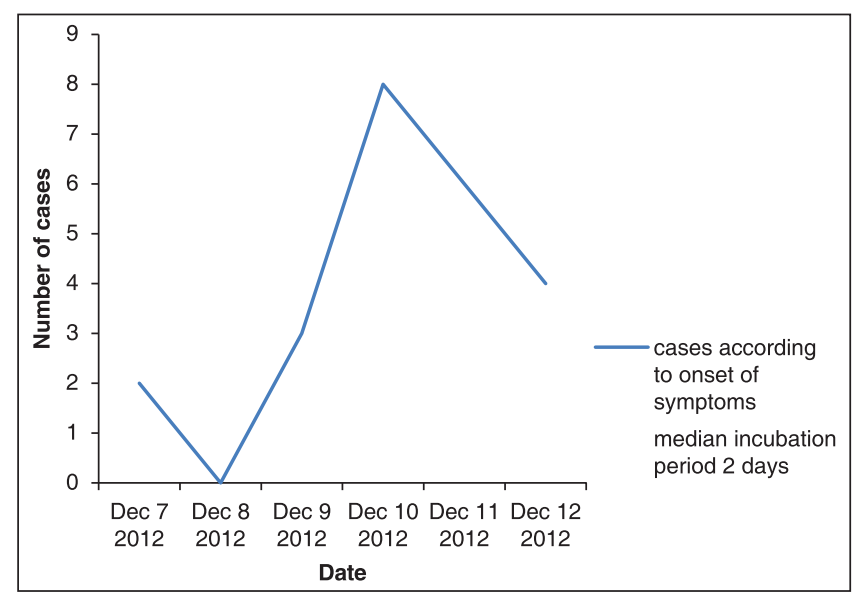

Figure 1: Number of cases according to onset of symptoms

Similar study conducted by Mustafa MS, "raita" was incriminated for food poisoning and when raita food sample was subjected to bacteriological analysis, $S$. aureus was isolated.

In the present outbreak, the results of investigation revealed that 'Kerala matta rice' was the food item responsible for occurrence of food poisoning. Persons who had consumed this rice item were 31 times more likely to become ill than those who did not consume 'Kerala matta rice'.

Staphylococcal enterotoxins induce food poisoning, when food contaminated with the enterotoxin produced by coagulase-positive Staphylococci is consumed. For staphylococcal food poisoning to occur, it is necessary that adequate time should have elapsed from the time of cooking to the time of consumption. Besides, optimum temperature must also be present for the bacteria to multiply and produce the enterotoxin..$^{[5]}$ Kerala matta rice was prepared in the morning at around 11.00 am and stored in the steel container at room temperature until mid-day, when it was served. Manual handling could have probably contaminated the rice with the staphylococci that had colonized in the nose and body surface of the food handlers. Another possibility is that rice could have been contaminated by the staphylococci present in the immediate surroundings such as steel container itself.

No samples like nasal swab were collected from food handlers to know if they were harboring Staphylococcus aureus. All food handlers in the private caterers were apparently healthy and showed no signs and symptoms of staphylococcal infection. Although food handlers are usually the main source of food contamination in food poisoning outbreaks, equipment and environmental surfaces can also be sources of contamination with S.aureus. ${ }^{[6]}$ Food handlers form an important link in the chain of any food poisoning outbreak.

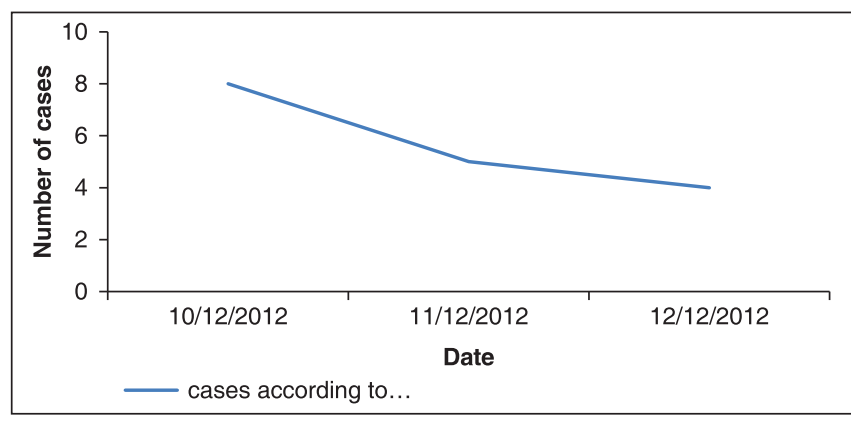

Figure 2: Number of cases according to admission

Hence they should be subjected to thorough clinical and laboratory evaluation to find out the probable source of infection.

Environmental factors that could have played an important role in bacterial proliferation and enterotoxin production are manual handling of cooked rice by the mess staff and storage of the rice at room temperature for a long time.

In order to reduce the incidence of staphylococcal food poisoning the food items should be prepared and served under hygienic conditions. Manual handling should be restricted to a minimum and food handlers should wash their hands thoroughly before handling food, after handling garbage and after visiting the toilet. Food contact surfaces and equipment such as knives, slicers and containers should be cleaned.

\section{REFERENCES}

1. Assuring food safety and quality: Guidelines for strengthening national food control systems. [online]. 2003 April [cited 2013 July 2]; Available from: http://www.who.int/foodsafety/publications/fs_management/guidelines_ foodcontrol/en/.

2. Mori M, Kato E, Hamada S. Distribution of enterotoxigenic staphylococci in healthy food handlers and biological properties of isolates. Japan J Bacteriol 1977;32:501-8.

3. Bergdoll MS. Staphylococcal food poisoning. In: Cliver DO, editor. Food borne Diseases. San Diego, California: Academic Press;1990. p. 85-106.

4. Adesiyun AA, Webb LA, Romain HT. Prevalence and characteristics of Staphylococcus aureus strains isolated from bulk and composite milk and cattle handlers. J Food Prot 1998;61:629-32.

5. Centers for disease control and prevention (CDC). Outbreak of staphylococcal food poisoning associated with precooked hamFlorida,1997. MMWR Morb Mortal Wkly Rep 1997;46:1189-91.

6. Loir YL, Baron F, Gautier M. Staphylococcus aureus and food poisoning. Genet Mol Res 2003;2:63-76.

How to cite this article: Basavegowda M, Rajegowda RM, Bettappa P, Shivanna UR, Channabasappa AN, Vijaykumar GS. An outbreak of staphylococcal food poisoning in a nursing hostel, Mysore, Karnataka. Int J Med Public Health 2014;4:257-9.

Source of Support: Nil, Conflict of Interest: None declared. 